\title{
ADVANCED 3D MODELING VERSUS BUILDING INFORMATION MODELING: THE CASE STUDY OF PALAZZO ETTOREO IN SACILE (ITALY)
}

\author{
D. Visintini ${ }^{1}$, E. Marcon ${ }^{1}$, G. Pantò ${ }^{1}$, E. P. Canevese ${ }^{2}$, T. De Gottardo ${ }^{2}$, I. Bertani ${ }^{3}$ \\ ${ }^{1}$ Polytechnic Department of Engineering and Architecture (DPIA), University of Udine, via delle Scienze 206, I-33100 Udine, Italy \\ - domenico.visintini@uniud.it; marcon.enrico.1@spes.uniud.it; giacomo.panto92@gmail.com \\ 2 Virtualgeo s.r.l., via Trento 105/D, I-33077 Sacile, Italy - (erminio.canevese, tiziano.degottardo)@ virtualgeo.it \\ ${ }^{3}$ Academy Virtualgeo s.r.1, via Trento 105/D, I-33077 Sacile, Italy - ilder.bertani@academyvg.com
}

KEY WORDS: Modeling, Advanced3D, BIM, HBIM, Scan-to-BIM, Comparison.

\begin{abstract}
:
This paper presents an experience of 3D modeling starting from laser scanning data and following two alternative approaches: the first one, called "Advanced 3D modeling", based on an original meshing algorithm, while the second make use of Revit BIM software. The case study in Palace Ettoreo in Sacile (Pordenone, Italy), constructed in Renaissance Venetian style in the 16th century: it has a trapezoid plan and is developed on three floors, with the ground one endowing a portico on two façades.

The palace has been surveyed by two terrestrial laser scanners: a Riegl Z420i for 5 external scans and a FARO Photon 120 for 53 internal scans; also a topographic surveying of 270 targets have been carried out. The final TLS cloud has 1,4 billions of points.

The Advanced 3D modeling has produced a "smart" mesh, allowing also to model the elements with deformations (out of plumb, bulges and troughs). Moreover, this model drastically reduce the stored data: the whole palace is modeled by 111.496 polygons only. The modeling with Revit follows the classical flowchart where the principal architectonical elements are gradually composed: this HBIM process has required a strong manual work in exploiting the available parametric objects and/or in the definition of new objects. Comparing the two models with respect the points cloud, both have evidenced advantages and limitations: therefore, the best solution is a process involving their combination. At the beginning, the Advanced 3D modeling is performed onto the points cloud, so well exploiting the segmentation tools and the smart meshing of the surfaces preserving any geometrical irregularity. Such obtained model allows metrical and morphological evaluation on the various structural and architectonical elements. Afterwards, this very light model becomes the entry data for the modeling in BIM environment, where also the shape of irregular elements are so imported.
\end{abstract}

\section{INTRODUCTION}

The 3D modeling of historical buildings is one of the goals and results of the Geomatics, when addressed to close-range terrestrial applications, in order to supply the knowledge geometric basis for any Restoration activities.

As well-known, nowadays Terrestrial Laser Scanning (TLS) and Structure from Motion (SfM) photogrammetric techniques allow to obtain, with complementary features compared e.g. in Gonizzi Barsanti et al. (2012), clouds of millions of 3D points. For these techniques, the most advanced (current) instrumentations are, respectively, images/video by drones for SfM photogrammetry, evaluated e.g. in Piras et al. (2017), and Indoor Mobile Mapping Systems (IMMS) for TLS, examined e.g. in Tucci et al. (2018). The achieved Level of Detail (LoD) and accuracy/precision are generally excellent, with a surveying efficacy/efficiency unthinkable just few years ago, also for very complex buildings. In other words, we can state that building 3D surveying is today fundamentally solved thanks to the modern geomatics techniques, often integrated, generating tons of $3 \mathrm{D}$ points.

The modeling of/from such clouds is instead an open problem, moreover concerning about the automation of such task; in any case, two different families of approaches can be pursued: the surface modeling and/or the object modeling, this last sometimes called solid modeling. In general, first 3D models were built by detailed surfaces as Dense Digital Surface Models (DDSM), often texturized by the acquired images, while currently the emerging idea is to obtain a model by suitably assembling single objects. In spite of this, both methodologies have pros/cons and the user, having software full availability, choices one or the other according to his own use of the model.

If the work objective is the BIM model of the building, with the consequent advantages of this choice, obviously the object modeling is mandatory: in this case, not only the building is modeled by means of single architectonical objects, but each one is parametric, either from the geometrical point of view or the material one. This application of the BIM process to an existing Historical/Heritage building is called HBIM, although other slightly different definitions of HBIM are reported in literature. A complete State-of-the-Art on HBIM schemes, surveying data acquisition, and modeling concepts is reported in Dore and Murphy (2017), while one notable HBIM model is the Basilica di Collemaggio (L'Aquila, Italy) reported in Brumana et al. (2018). The Scan-to-BIM process is therefore the articulated phase where the TLS (but also photogrammetric) clouds become BIM objects, with more or less complex operations carried out by the modeler. In such process, some fundamental index of quality of the obtained model have to be taken into account: following Banfi (2017), evaluating the Grade of Generation (GoG), the Grade of Information (GoI) and the Grade of Accuracy (GoA), a specific model (or its part) can/cannot be used in a certain BIM analysis. Coming back to the modeling aspect, the aim of this work is the comparison of the surface modeling versus the BIM modeling of a TLS points cloud of a 16th century palace. Obviously, the Scanto-DSM processing is not a Scan-to-BIM process, but anyway the two obtained models can be compared, making use of quantities similar to those applied for evaluating the GoA of a BIM model.

\section{SURVEYING DATA ACQUISITION}

\subsection{Case study}

Palazzo Ettoreo was constructed in Sacile (Pordenone, Italy) in the mid- $16^{\text {th }}$ century on a previously Gothic-style building, as a noble house with the elegantly sober appearance of the Renaissance Venetian architecture. In that period, in fact, Sacile lived its maximum economic expense, earning the nickname of "Garden of Venice". The palace has a trapezoid plan, developed on three floors, with the ground one endowing a portico on two façades, a third side faces an internal courtyard, while the fourth 
divides and connects the palace to the near building. Numerous frescoes decorated the façades in the past, of which now only traces remain: three cherubs, which probably completed a complex decoration, and a fragment in a round frame underneath the portico depicting a Madonna and Child. The portico consists on one side of three elegant round arches on octagonal stone columns and, in the other, of six brick arches enclosing the original columns. In the inner courtyard, an impressive staircase leads to the large noble salon; this room has a ceiling with decorated wooden beams, a suggestive angular window and a beautiful four-arched window with balcony in Istrian stone.

The building, now owned by the City Council, was home to the "Golden Lion" guesthouse for over a century: Silvio Pellico stayed there on 25th March 1822, while moving from Venice to the Spielberg prison in Brno (Czech Republic).

\subsection{TLS surveying}

Palazzo Ettoreo has been surveyed by TLS techniques, anyway exploiting, as recommended, a topographic surveying in order to fix the $3 \mathrm{D}$ position of the targets used in the scans registration. A Leica TCRA 1201 total station has been employed to define a principal topographic network, on which ten polygonal chains were constrained. Therefore, from so estimated 53 polygonal vertexes, 270 targets were measured.

In the meantime, the palace was surveyed by employing two TLS instruments: the time-of-flight Riegl Z420i, acquiring 5 external scans, and the phase difference FARO Photon 120, used instead for acquiring 53 internal scans.

By exploiting the topographic 3D coordinates of the targets, all the scans have been so registered and geo-referenced at the same time. The final TLS cloud has 1,4 billions of points (Figure 1), whose file requires a storage size of $1,35 \mathrm{~GB}$ !

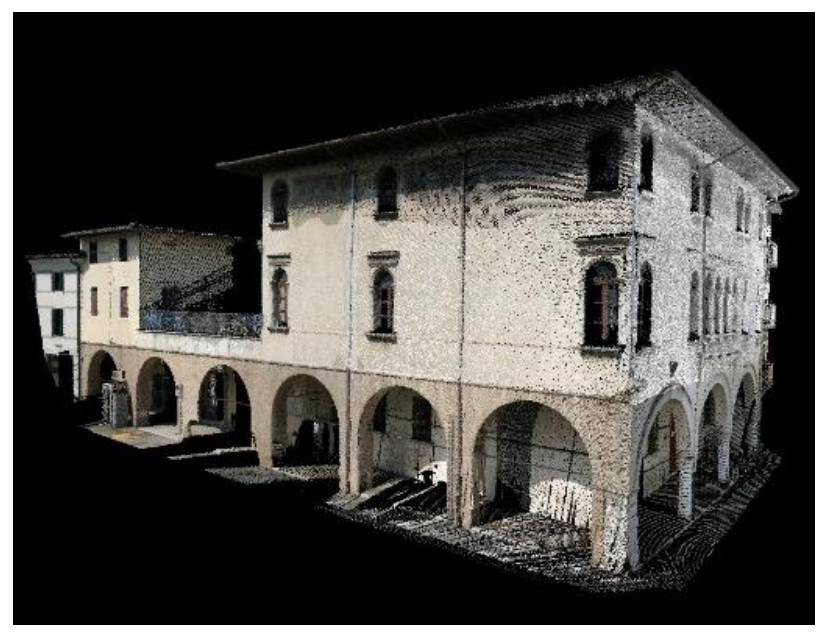

Figure 1. Acquired TLS points cloud.

\section{ADVANCED 3D MODELING}

\subsection{Definition}

The term "Advanced 3D" recalls the fulfilments of this modeling method to the European Commission's Reflective 7 directive "Advanced 3D modelling for accessing and understanding European cultural assets" of the Horizon 2020 project. Some details on this meshing algorithm will be later explained, but the basic idea is to iteratively build a quadrangular surface, locally increasing the number of faces, then reducing their sizes, until all vertexes have a distance from the TLS (or photogrammetric) points cloud lower than a fixed threshold. This value of maximum acceptable distance defines a sort of "modeling simplification" index, very similar to the GoA value used instead to evaluate HBIM models. The final obtained "smart" mesh makes so possible to identify the different architectonical elements, as well as to improve the modeling details for the elements with significant irregularities and deformations (out of plumb, bulges and troughs). Furthermore, parts of the segmented model can be linked to specific information, e.g. degradation data, as done for the river port of Aquileia (Italy) and described in Pratali Maffei et al. (2019).

A so-obtained Advanced 3D (A3D) model could have different types of development involving geometry, texture and information. Considering an A3D model obtained from TLS clouds, since it preserves all the acquired geometric data (within the previous simplification threshold) it can be defined as a "Lidar Information Model" (LIM). If such model is also texturized, suitably exploiting well-known photogrammetric rules and software tools, it can be called "Photo 3D Model" (PDM). More details about LIM and PDM definitions can be found in Canevese and De Gottardo (2019).

In the Palazzo Ettoreo case study, we will use a LIM model without any info data linked, so the GoI is purely geometric.

\subsection{Cloud segmentation}

First of all, the TLS points cloud is divided in smaller portions by means of EasyCUBE Virtualgeo software, by manually sharing external façades form inner points, these lasts in rooms and, for each room, among single walls, floors and ceilings, up to distinguish single architectural units.

The modeler chooses the level of such segmentation, in accordance to the project manager requirements. Each portion is assigned to a different layer and is cleaned of all the data not related to the target element, namely obstacles or stranger bodies acquired during the surveying such as people, trees, furniture. Which points are deleted is, again, a modeler decision.

\subsection{Smart quad-surface modeling}

This is the fundamental step in obtaining an A3D model: the modeler selects a suitable point of view, logically as much as possible in front of the segmented cloud, and fixes a first attempt "large" dimension of the grid. Furthermore, he fixes the maximum deviation acceptable between the final quad-surface and the points in the segmented cloud. This parameter is derived form a design intent, that the modeler need to take form the project manager. In alternative, modeler can push the model to the limit to give the best reliability possible, nevertheless taking into account the cloud noise, at least due to the merging different scans. The grid points are projected to the cloud point and the vertexes bonded to the cloud. The quad-surface is then applied to the vertexes: this surface can be called "smart" because if the deviation of the quad-surface from the cloud points is greater than the modeler threshold, then the grid is locally divided in smaller pieces. This iterative process is fully automatic: at the end, the data amount is drastically reduced since the obtained LIM data, preserving all the "relevant" geometric information, can be used instead of the cloud. Since the modeler has the full control of each A3D step, we can say that the obtained model has a numerical and realistic value of GoA (albeit is not a BIM model).

\subsection{TIN details modeling}

When the architectural element is very small, has a very irregular shape or the modeler decides that every geometric detail have to be take into account, the quad-surface is not the best tool to be used. EasyCUBE software makes available various tools to generate usual TIN (Triangulated Irregular Network) meshes, based on the well-known Delaunay triangulation, and to 
edit/improve them, by smoothing, closing holes, decreasing polygons, again within a maximum deviation from TLS cloud.

\subsection{TIN modeling}

If the modeler decides instead to not follow the A3D modeling algorithm by fixing a threshold value, EasyCUBE allows to model all the segmented clouds using standard TIN procedures, combined with commands of extrusion, loft and similar, obviously with the same editing tools described before.

\subsection{Assembly and features}

At the end of the modeling process, each obtained surface, be it a quad-surface or a mesh, has to be related to the near ones. The modeler can extend, trim or chamfer each mesh, so automatically generating the edges between elements.

The so obtained A3D model of the palace has 216 layers, 111.496 polygons and a size of $6,6 \mathrm{MB}$ only (while the TLS cloud size was 1,35 GB) and appears as shown in Figure 2; different colours means different aggregate elements of the building.

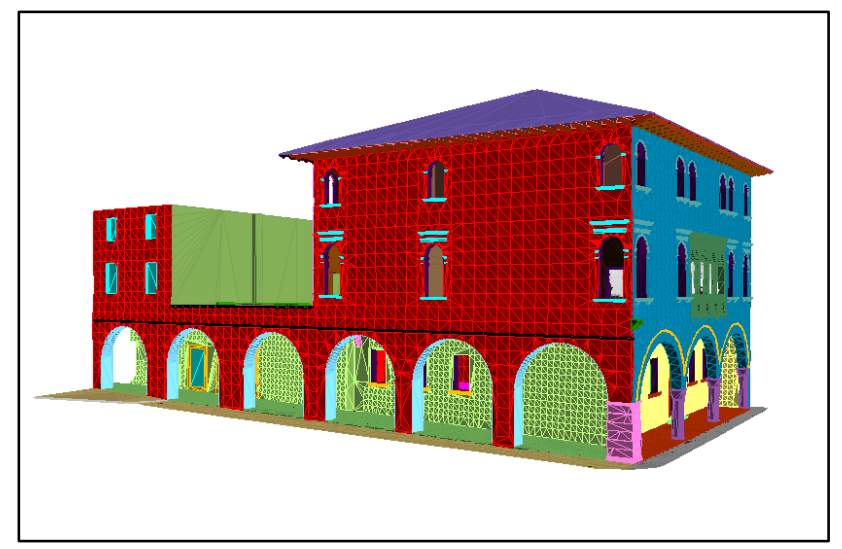

Figure 2. Advanced 3D model obtained by EasyCUBE.

\section{BUILDING INFORMATION MODELING}

Starting from the same TLS cloud, the Scan-to-BIM process has been later carried out, so to produce the HBIM model of palace. For such aim, two students graduating in Master Degree Course in Architecture, then well-knowing constructive techniques but without experience in BIM authoring tools, team up with an expert on Autodesk Revit and developer of parametric families. The goal was not to specific use of Revit, but to translate the students knowledge and workflow to a BIM authoring tool.

\subsection{Project setup}

Although the standard process begins from a TLS cloud, here the previous A3D LIM model is instead exploited and, by available importing tools, charged into the Revit 2019 environment.

As usual in BIM software, also Revit requires the definition of the building levels, where each object is hosted. Hence, the first project setup is to define how many levels are to be placed: because a level is defined by an elevation, we decide to put levels on top of each floor and another where the roof meets walls.

\subsection{Walls modeling}

In principle, there are multiple ways to model this building in Revit, but we decide to start using only standard tools, even if we know that not one wall is perfectly vertical: this is one of the stronger modeling limitations following a BIM approach.
The first problem in tracing a standard wall on a point cloud is which points must be selected. Using A3D model as a reference, we have not so many points to consider. The problem become easier, but not already solved at all because the A3D model shows wall surfaces are not vertical. This situation, occurred for many walls of this $16^{\text {th }}$ century palace and moreover frequent for older heritage buildings, has the logical consequence that points on wall top don't match in planimetry with the points at the bottom. Using instead points at $1,10 \mathrm{~m}$ from the floor, as conventionally adopted to obtain an architectural plan as horizontal section of walls (doors and windows), even these points don't match with the previous. So, how many points and at which elevation we need? The real problem is that, in BIM software, there is no threshold, no maximum distance between model and input data. In general, no smart tools for wall construction are available, and modeler has to follow a "try and error" procedure and, as consequence, the model GoA cannot be known a priori but become evaluable only a posteriori on the obtained model.

Regarding the materials data of the walls, since at this time we don't know the internal composition, a first "generic material" for the core, and a second "generic material" for the layers packing it, are assumed (Figure 3). In this way, we can change finishes wall materials without changing all the wall definition, stating explicitly our current GoI to whoever will use our model.

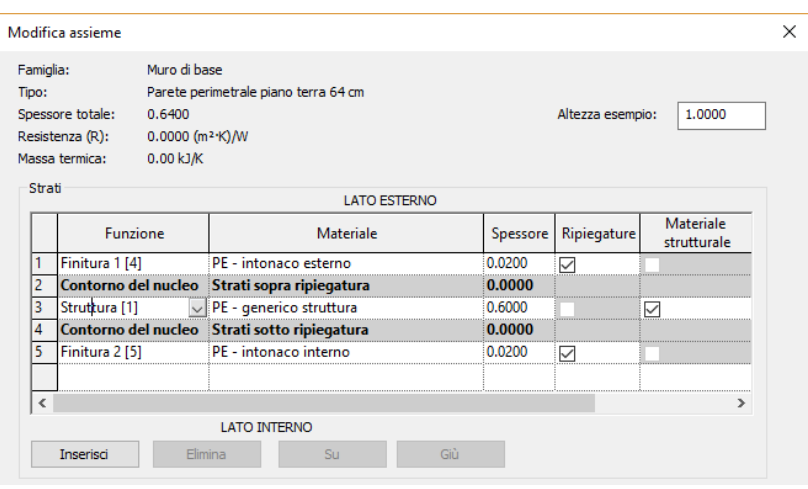

Figure 3. Example of wall definition in Revit.

\subsection{Portico arch modeling}

The first use of a "parametric family" is required for the modeling of the portico arch on the front. Even if there is in Revit a family developer, the parameters and requirements are stated by the students. The arising problem is that arch imposts are not exactly at the same elevation, but students don't realize it from LIM model. In this way, trimming the arch side to make the molding continuous, the arch model fails at the column capital (Figure 4).

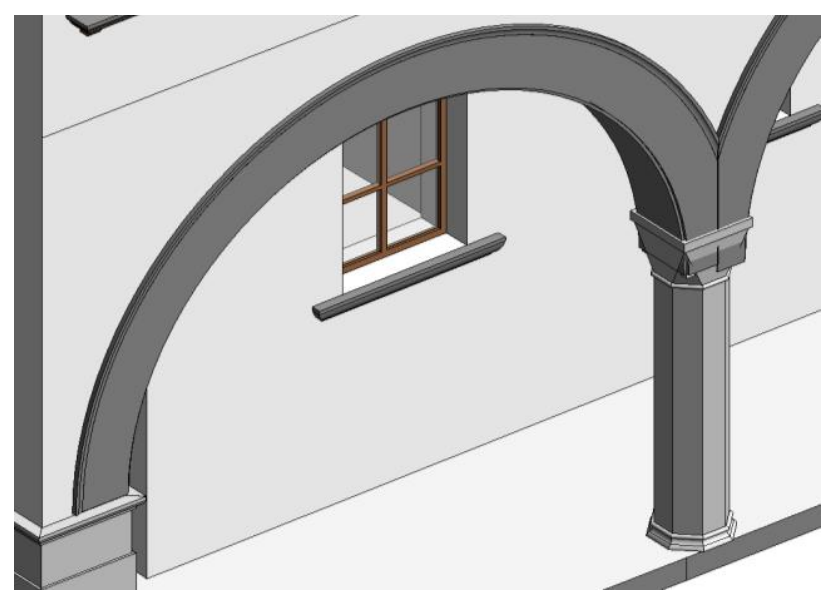

Figure 4. Modeling problems at column capital (at right). 
This lesson learned by students point out the importance of requirements and parameters for this family, and clearly for any other element e.g. the windows, growing the modeling precision.

\subsection{Portico columns modeling}

Modeling portico columns, a new decision has to be taken in order to define this "element" or, in other words, the architectural syntax used to describe this part of building. Is the column considered a single element or must be divided in base, shaft and capital? How many parameters are needed to describe this element? Further than geometric syntax, also materials, codes lexicon to describe degradation (UNI 11182/2006), parameters to describe restoration works and so on should to be defined. The decision is pivotal because, not only change the modelling technique (nested shared families vs simple families), but also change the information container and the parameters involved in the information storage, namely the Level of Information (LoI). Another fundamental aspect of the model is its Level of Detail (LoD). In our case, some columns capital are decorated, some windows have peculiar elements: such data have to be modeled or not? Only the Project Manager can decide the "right" LoD for a certain HBIM model, because he is the one who decide the design intent of the whole restoration project.

\subsection{Floors, ceilings and roof modeling}

We use standard Revit "floor tools" with generic materials to model all the floors at each level, nevertheless knowing that for a historical building floor to be planar and horizontal is not an assumption, rather it's a mistake.

Similar drastic simplification is made using the "structural beam tool" to model the ceiling wooden beams, losing their vertical deflection and torsional displacements. This BIM modeling restriction for an architectural but also structural element as the wooden beams, that could be extremely irregular, is one of the main bottleneck for HBIM applications, also considering to perform resistance analyses with structural plug-ins.

Modeling roofs is moreover difficult, further than TLS surveying troubles, because the ridges and the gutters are not horizontal and not parallels, while the roof fields are not planes.

\subsection{Stairs modeling}

Modeling stairs is always a complex task, and also in Revit this is a bit complicated. Internal staircases are modelled by the available "stair tools" by components, while for the yard stair we implemented the stair tool "by drawing" to align threads that have all different extension to their position.

We also use a parametric family to model balusters that have a peculiar form and different dimensions.

\subsection{Assembly and features}

All the previous elements modeled in Revit (eventually joined) have automatic intersections, based on the classification of the element, the function of the layer and the construction material associated. When intersections have multiple solution, software asks to the modeler to decide which solution is the right one. In our specific model, having used generic materials, this interesting feature is not used at its full capability: only in some cases, e.g. where three or more walls depart from a single edge, we have implemented a specific intersection solution.

The final complete Revit HBIM model is depicted in Figure 5.

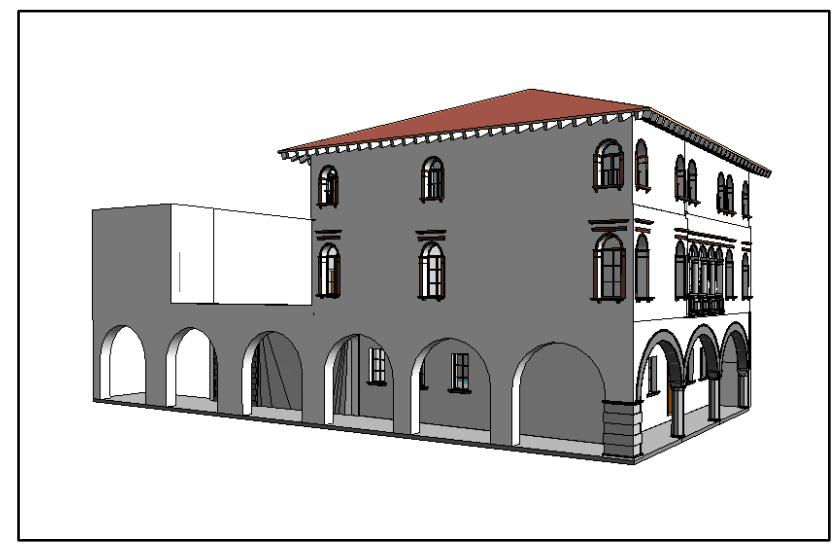

Figure 5. HBIM model obtained by Revit.

\section{COMPARISON A3D VS HBIM VS TLS}

To geometrically compare the two previous models to the TLS points cloud, we use some tools in EasyCUBE allowing such computations and giving coloured representations.

Differences A3D vs TLS give us a validation of the meshing process and the fulfilment of distance assumed as threshold.

For the HBIM vs TLS ones, these can be interpreted as the final effect of the propagation of various error due, al least, to using BIM standard tools, tracing elements manually, choosing a low LoD. In other words, these represent the model GoA.

For the comparison, we select three elements with different shapes of the palace: the second run of the inner yard stair that connects the yard with the noble salon, one of the long walls of this room, and the greatest exterior wall. Such architectonical element as highlighted in blue in Figure 6.

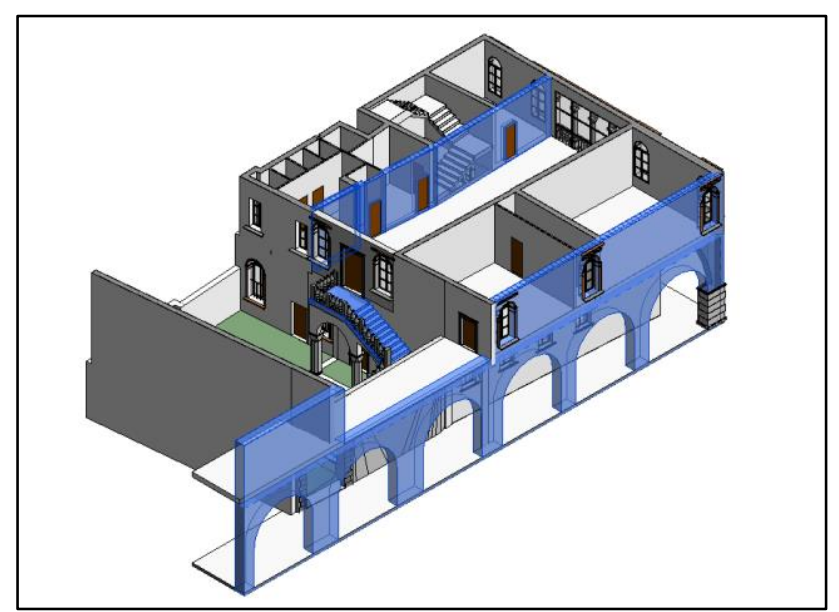

Figure 6. Elements chosen for comparison.

From now on, the subsequent figures show each part of the evaluated element coloured according to the local distance with respect the TLS cloud. Best results are depicted in blue, meaning a $0-1 \mathrm{~cm}$ (in absolute value) deviation, turning in green for 1-2 $\mathrm{cm}$ values, then becoming yellow orange and red for $2-3 \mathrm{~cm}$. Surface coloured by black corresponds to discrepancies out of this $3 \mathrm{~cm}$ range. If it happens in the element borders, it can be a lack of data and then not significant, while occurring in the central parts, it means a gross A3D/HBIM modeling error. 


\subsection{Stair}

The segmented cloud contains about 1.000 .000 points and it is noticeable the large hole (lack of data) where the TLS instrument have been placed during the surveying.

Figure 7 compares A3D model to the segmented TLS point cloud: as one can see, the main values is around $0,5 \mathrm{~cm}$.

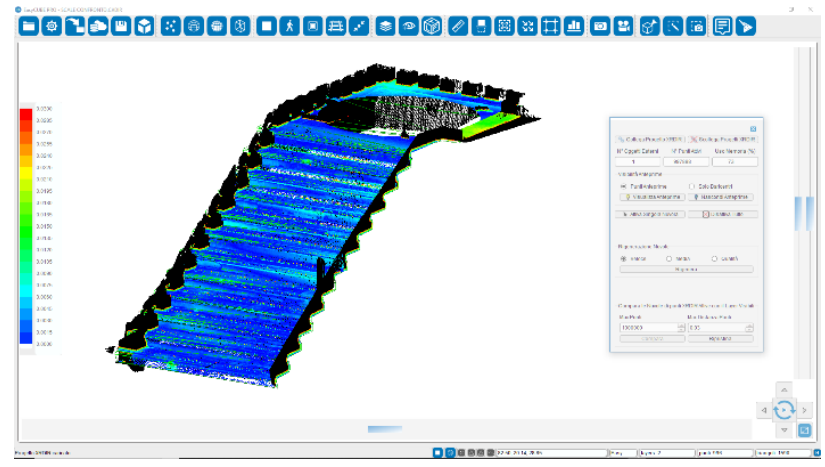

Figure 7. Stair: Advanced 3D model vs TLS points cloud.

Figure 8 shows instead comparison between HBIM model and the same segmented TLS point cloud. The results are worse: as told before, we have used the stair tool "by drawing" in Revit and, doing so, we have decided one value for the risers height, but the last two rises seems to have different heights. Another error is in the thread lines that seems not perpendicular to the stair path. These results confirm that, if the design intent requires an HBIM model with better reliability, the standard tool must be left aside and other more complex Revit tools, such as "conceptual mass" or "adaptive families", have to be used.

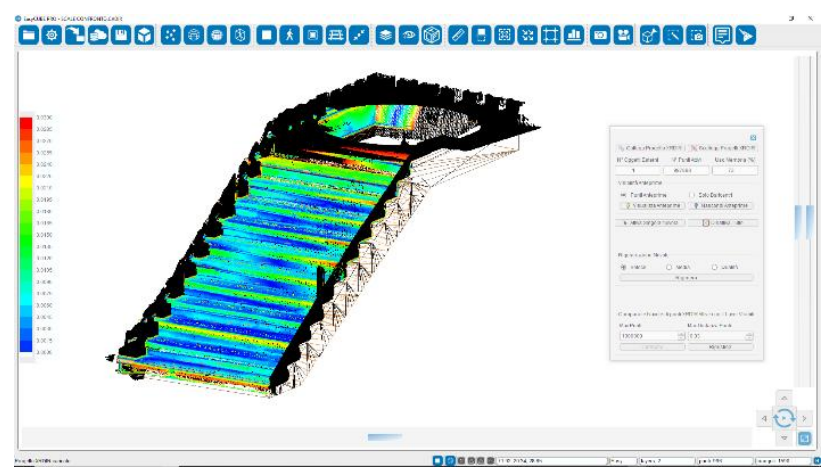

Figure 8. Stair: HBIM model vs TLS points cloud.

\subsection{Internal wall}

From the prevalent blue colouring the wall shown in Figure 9, one can see that smart quad-surface and point cloud are nearly interchangeable, since mean differences are again around $0,5 \mathrm{~cm}$.

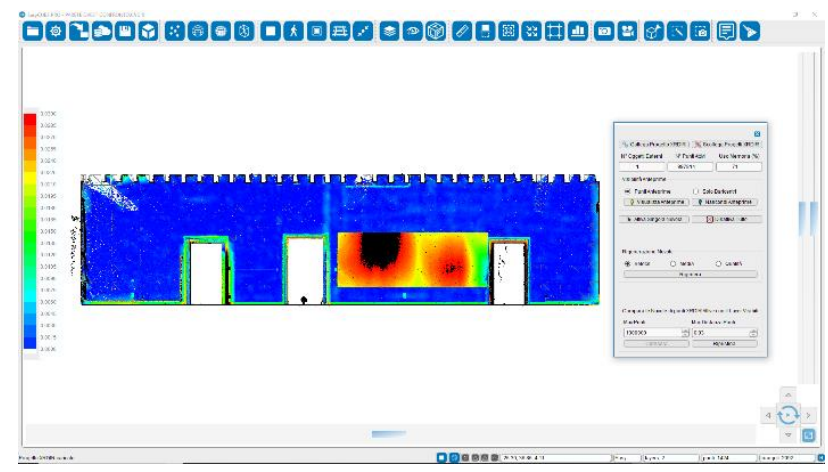

Figure 9. Internal wall: Advanced 3D vs TLS points cloud.
Red and also black coloured element between doors is an accessory applied to the wall, removed in A3D modeling, while kept in the reference TLS cloud, left as acquired. The doors chasing and panels are instead not selected for the comparison.

Figure 10 shows that BIM model, a part in the accessory area, has some issues: on the right end, there is a problem with the points taken to trace the walls, maybe they are too few and very noised. This wall must be modelled again. Considering now the left side: over the first door, black colour means that the wall has a strong off-plumb, over $3 \mathrm{~cm}$; changing colours along horizontal states an azimuthal irregularity, not modeled by the Revit tools utilized.

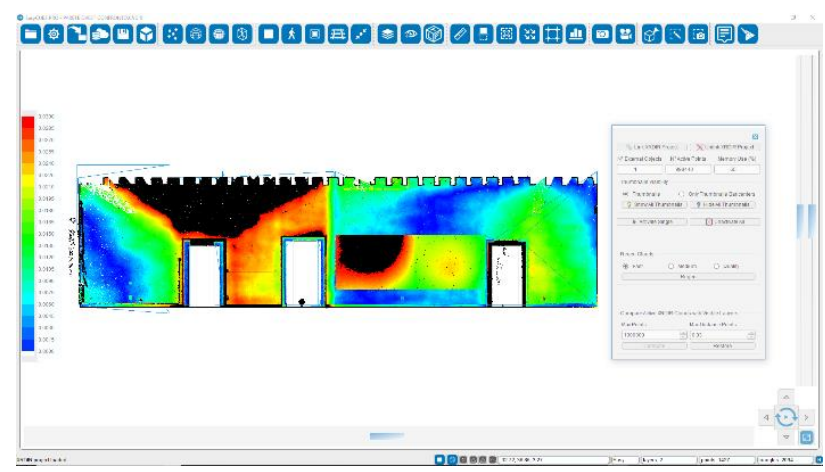

Figure 10. Internal wall: HBIM vs TLS points cloud.

\subsection{External wall}

This wall has a very large horizontal extension and vertically goes form the portico floor to the roof: it looks as ideal to test the behaviour of the two modeling approaches when a large element of an historical, then probably irregular, building is involved. From the colour map shown in Figure 11, again mainly presenting blue graduations, it is numerically evidenced again as the smart A3D quad-surface and point cloud are very similar.

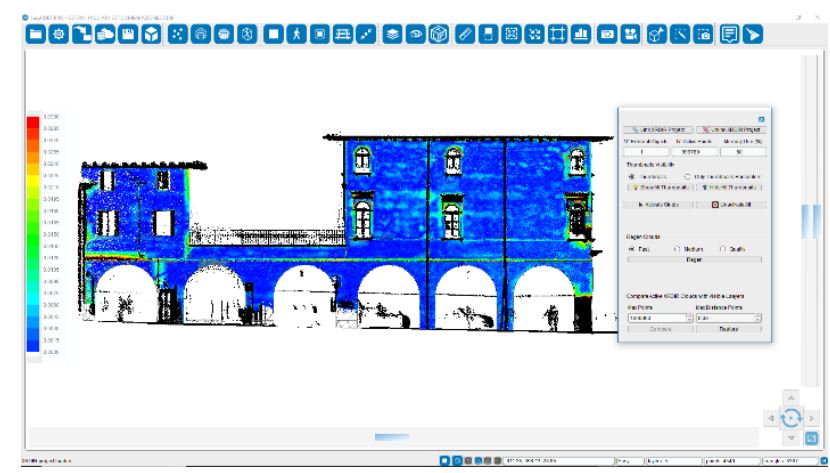

Figure 11. External wall: Advanced 3D vs TLS points cloud.

The comparison of HBIM model surfaces, represented in Figure 12 , shows again some defects in wall modelling.

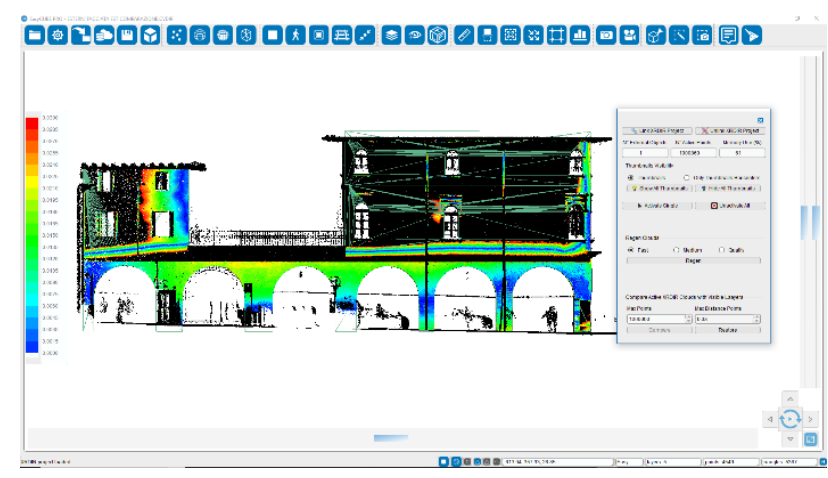

Figure 12. External wall: HBIM vs TLS points cloud. 
Using parametric wall objects, having vertical planes, already at the first floor, the absolute deviation is out of $3 \mathrm{~cm}$ range.

One possible cause is that the wall has been vertically modelled too far from the TLS cloud and should to be, at least, shifted towards the inner. Further investigations are surely required. Anyway, we have here extended the colour range up to $0-15 \mathrm{~cm}$. In Figure 13 is shown the same comparison, but now colour graduation is blue for $0-3 \mathrm{~cm}$ deviation, green for $3-9 \mathrm{~cm}$, yellow orange and red for $9-15 \mathrm{~cm}$ and, again, black means out of range.

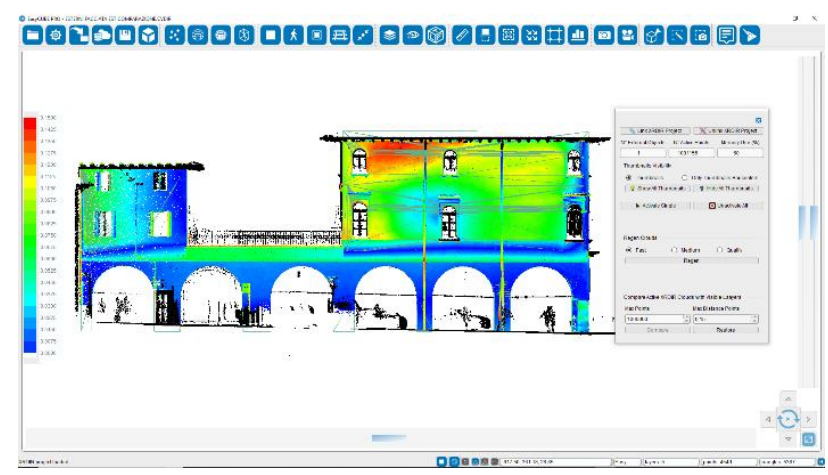

Figure 13. External wall: new scale colours for HBIM vs TLS.

Cian region in the first column ad right is due to a stone cover that hasn't been selected for comparison: in truth, such part was out of range also for $\mathrm{A} 3 \mathrm{D}$ model. Observing the whole façade from the first floor to the roof, there are a lot of colour changes: it means that such external wall is not only out of plane (not vertical) but also very irregular in form.

\section{OVERALL EVALUATIONS}

Before to asses the differences between the A3D and HBIM global models, a surely positive opportunity is the integrated use of both in the Revit environment. All the representation tools, efficiently producing plans, sections, elevations, 3D views, etc. make further modeling a lot easier taking distances or adding dimensions. Figure 14 shows together A3D and HBIM models.

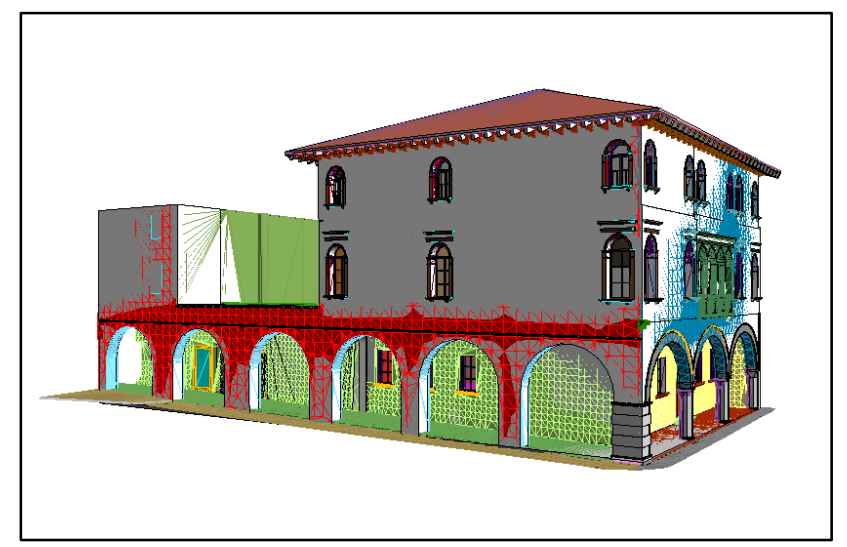

Figure 14. Fusion of Advanced 3D and BIM models.

Beginning the evaluations, as already stated and here recalled, an A3D model and a BIM model have different approach and roles. An A3D model is reliable and thousand times lighter in size than the acquired TLS point cloud: at least, this means there is no need of top notch computers. Even more, elements segmented in different layers can help reading the architectural structure and components of the building and can be a starting base for other models. It can be used for metrology analysis, such as deflection or torsion evaluation. Figure 15 shows a graphical representation of the irregular shape of the external walls, tracing on the wall surface curves with the same deviation from a vertical reference plane. Although not visible in figure, each curve is labelled with the corresponding deviation. Such detailed metrological data are fundamental as "zero epoch" situation for any possible analysis of the variations along the time (monitoring).

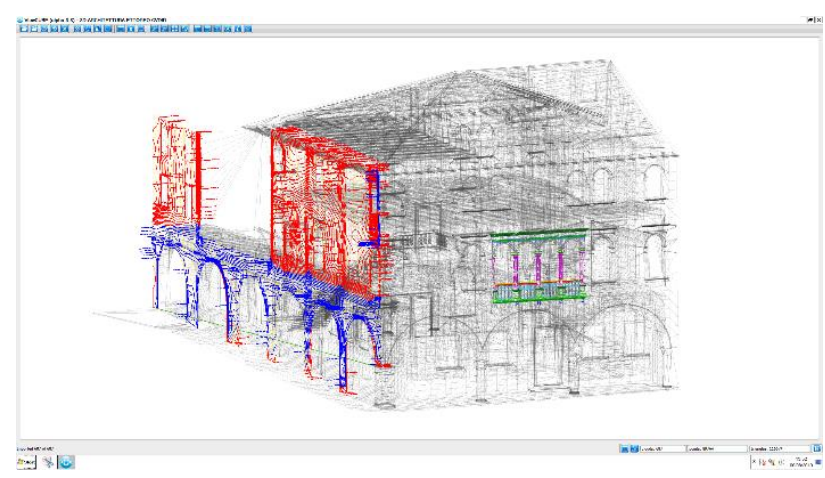

Figure 15. Advanced 3D model off plane curves.

Used for survey deterioration types and instances, it can be used in restoration projects and preservation management.

Being a surface description of the building, it has not data structure other than layers to identify building elements. Not being a solid model and without the data structure for building materials or intersection priority, documentation such as sections and floor plans must be hugely post-processed and improved by the modeler, in order to be represented with drawing standards.

Summarizing, the main advantage of using A3D/LIM model instead of point cloud is the easiness in model management since there are no more millions/billions of points to be evaluated: actually there are no more points at all, no more cloud noise nor external objects surveyed. The approach drawback is given from the simplification error, since A3D model and points cloud are different but, by direct comparison A3D vs TLS, this error has been evaluate in the order of a few millimeters.

As known, an HBIM model is assembled instead with building elements, and a lot of optimized tools are available to collect and manage information, make analysis, create documentation at easy, etc. Nonetheless, new tools specific for each building element should be implemented, so to improve software and design efficiency. From the results of the paper case study, the tools and data structure available in Revit may result a heavy constrain in the modeling and, to bypass them, advanced techniques are needed. In truth, effort to make a HBIM model reliable is often not justified by the cost of software developing. A logical solution in the evaluation of A3D vs HBIM models is that represented in Figure 16, where their integrated use is shown.

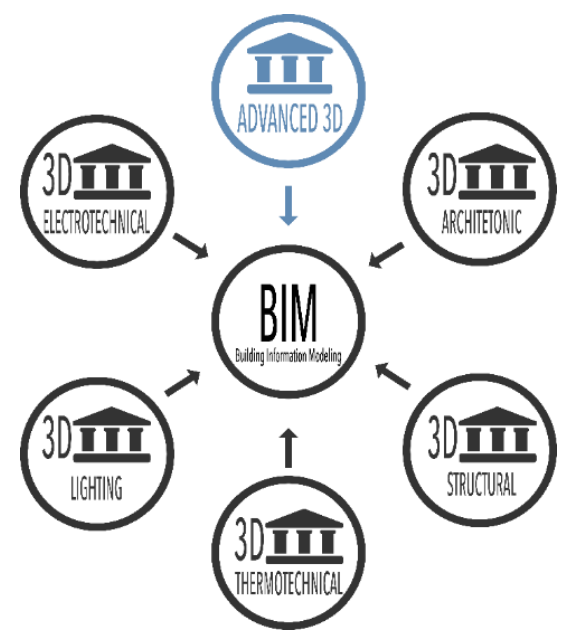

Figure 16. Advanced 3D as part of BIM data environment. 
All BIM analyses (architectonic, structural, thermotechnical, etc.) involves the same BIM/HBIM model, but the syntax used e.g. by the structural software is different form the description of the same element used in bill of quantities or sustainability and energy analysis. Even architectural models of the same building can have huge differences because of a different design intent used to make them. An A3D model can be hence exploited as a new component of the BIM common data environment.

\section{CONCLUSIONS}

Throughout the case study of the 3D modeling of Palazzo Ettoreo in Sacile starting from laser scanning data, two modeling approaches have been pursued: one is named Advanced 3D (surface oriented), the other is a common BIM (object oriented). After different and specifics steps, both gave as output a 3D model characterized by advantages and limitations.

Already in the modeling phases and even more in the comparison of the two models, the expression "Advanced 3D versus HBIM" loses its combative meaning, since it is clear that they can definitely cooperate and the better expression "Advanced 3D and HBIM" is the logical substitution.

The Advanced 3D modeling can be applied on the original TLS points cloud, exploiting segmentation tools and, above all, automatically creating a smart quad-surface, anyway preserving each geometrical irregularity. Such obtained mesh allows fine metrical and morphological evaluation on the various structural and architectonical elements. Subsequently, this very light polygonal model becomes the entry data, instead of the original cloud, for the modeling in BIM environment: in this way, also the geometry of irregular elements are imported as meshes.

This integrated approach makes possible a simplification in the construction of ad-hoc BIM objects, since their specific geometry is already defined. In other words, in the so obtained HBIM model can be stored further the geometric data, also all the particular material features of an historical building.

\section{REFERENCES}

Banfi, F., 2017. BIM orientation: Grades of Generation and Information for different type of analysis and management process. The International Archives of the Photogrammetry, Remote Sensing and Spatial Information Sciences, XLII-2/W5, pp. 57-64.

Brumana, R., Della Torre, S., Previtali, M., Barazzetti, L., Cantini, L., Oreni, D., Banfi, F., 2018. Generative HBIM modelling to embody complexity (LOD, LOG, LOA, LOI): surveying, preservation, site intervention - the Basilica di Collemaggio (L'Aquila). Applied Geomatics, 10(4), pp. 545-567.

Canevese, E.P., De Gottardo, T., 2017. Beyond point clouds and virtual reality: Innovative methods and technologies for the protection and promotion of cultural heritage. The International Archives of the Photogrammetry, Remote Sensing and Spatial Information Sciences, XLII-5/W1, pp. 685-691.

Dore, C., Murphy, M., 2017. Current state of the art historic building information modelling. The International Archives of the Photogrammetry, Remote Sensing and Spatial Information Sciences, XLII-2/W5, pp. 185-192.

Gonizzi Barsanti, S., Remondino, F., Visintini, D., 2012. Photogrammetry and laser scanning for archaeological site 3D modeling - some critical issues. Proceeding of the 2nd Workshop on "The New Technologies for Aquileia", 948, pp. B1-B10.
Piras, M., Di Pietra V., Visintini D., 2017. 3D Modeling of industrial heritage building using COTSs system: test, limits and performances. The International Archives of the Photogrammetry, Remote Sensing and Spatial Information Sciences, XLII-2/W6, pp. 281-288.

Pratali Maffei, S., Canevese, E.P., De Gottardo, T., 2019. The real in the virtual. The 3D model in the cultural heritage sector: the tip of the iceberg. The International Archives of the Photogrammetry, Remote Sensing and Spatial Information Sciences, XLII-2/W9, pp. 615-621.

Tucci, G., Visintini, D., Bonora, V., Parisi, E., 2018. Examination of Indoor Mobile Mapping Systems in a diversified internal/external test field. Applied Sciences, 8, pp. 401-430. 